Gut and Liver, Vol. 11, No. 5, September 2017, pp. 684-692

\title{
Nomograms to Predict the Individual Survival of Patients with Solitary Hepatocellular Carcinoma after Hepatectomy
}

\author{
Junyi Shen ${ }^{1}$, Linye $\mathrm{He}^{1}$, Chuan $\mathrm{Li}^{1}$, Tianfu Wen ${ }^{1}$, Weixia Chen ${ }^{2}$, Changli $\mathrm{Lu}^{3}$, Lvnan $\mathrm{Yan}^{1}$, Bo Li ${ }^{1}$, and Jiayin Yang ${ }^{1}$ \\ ${ }^{1}$ Department of Liver Surgery \& Liver Transplantation Center, Departments of ${ }^{2}$ Radiology and ${ }^{3}$ Pathology, West China Hospital, Sichuan \\ University, Chengdu, China
}

Background/Aims: Solitary hepatocellular carcinoma (HCC) is a subgroup of HCCs. We aimed to establish nomograms for predicting the survival of solitary HCC patients after hepatectomy. Methods: A total of 538 solitary HCC patients were randomly classified into training and validation sets. A Cox model was used to identify predictors of overall survival (OS) in the training set. A nomogram was generated based on these predictors and was validated using the validation set. Results: Tumor size, microvascular invasion, and major vascular invasion were significantly associated with OS in the training set. Nomograms were developed based on these predictors in the multivariate analysis. The $\mathrm{C}$-index was 0.75 for the OS nomogram and 0.72 for the recurrence-free survival nomogram. Compared to the index of conventional staging systems for predicting survival ( 0.71 for Barcelona Clinic Liver Cancer, 0.66 for the seventh American Joint Committee on Cancer, 0.68 for Cancer of the Liver Italian Program, and 0.70 for Hong Kong Liver Cancer), the index of the OS nomogram was significantly higher. Moreover, the calibration curve fitted well between the predicted and observed survival rate. Similarly, in the validation set, the nomogram discrimination was superior to those of the four staging systems $(p<0.001)$. Conclusions: The nomograms demonstrated good discrimination performance in predicting 3- and 5-year survival rates for solitary HCCs after hepatectomy. (Gut Liver 2017;11:684692)

Key Words: Nomograms; Prognosis; Carcinoma, hepatocellular; Surgery

\section{INTRODUCTION}

Hepatocellular carcinoma (HCC) has been the third most com- mon cause of cancer-related death worldwide. Hepatitis B viral infection has resulted in high presence of HCC in china. ${ }^{1,2}$ Solitary HCCs is subgroup of HCCs. According to the recent Barcelona Clinic Liver Cancer (BCLC) staging system, solitary HCCs are classified as BCLC A regardless of the presence of microvascular invasion (MVI). ${ }^{3}$ According to the seventh American Joint Committee on Cancer (AJCC) staging system, solitary HCCs are classified as tumor stage 1 or 2 regardless of tumor size. ${ }^{4}$ Hong Kong Liver Cancer (HKLC) staging system classify single tumor into early or intermediate tumor based on tumor size. ${ }^{5}$ There is no consensus on the stage of solitary HCCs. Solitary HCCs include heterogeneous tumors because HCC has high presence of adverse clinicopathological factors affecting prognosis, such as vascular invasion, when the tumor size increases. ${ }^{6-8}$ Vascular invasion had been validated as an important prognostic factor. Some solitary HCC patients had, while some had not. ${ }^{9-12}$ In the past few decades, technical improvements in hepatic resection and perioperative managements, some large HCC $(>5 \mathrm{~cm})$ could be effectively and safely applied for hepatectomy. ${ }^{13-15}$ However, the relationship between tumor size and prognosis after curative resection remains unclear for solitary HCCs. The majority of studies reported patients with large HCC have poorer long-term outcomes than those with small HCC after curative resection. ${ }^{16-18}$ A few studies showed that patients with large HCC have similar outcomes to those with small HCC., ${ }^{9,19}$

The BCLC staging system and the HKLC staging system offer both management guidance and prognostic prediction., ${ }^{3,5}$ The seventh AJCC edition and Cancer of the Liver Italian Program (CLIP) also had distinct prognostic performance. ${ }^{4,20}$ These conventional staging systems have some limitations because they might not take full clinicopathological predictors into account when stratifying the prognosis. For example, few staging systems, except for the seventh AJCC staging system, emphasize

Correspondence to: Tianfu Wen

Department of Liver Surgery \& Liver Transplantation Center, West China Hospital, Sichuan University, No. 37 Guo Xue Xiang, Chengdu 610041, China

Tel: +86-18980601471, Fax: +86-02885422055, E-mail: cdwentianfu@126.com

Received on September 15, 2016. Revised on November 10, 2016. Accepted on January 9, 2017. Published online June 27, 2017 pISSN 1976-2283 eISSN 2005-1212 https://doi.org/10.5009/gnl16465

@ This is an Open Access article distributed under the terms of the Creative Commons Attribution Non-Commercial License (http://creativecommons.org/licenses/by-nc/4.0) which permits unrestricted non-commercial use, distribution, and reproduction in any medium, provided the original work is properly cited. 
the role of MVI. Besides, these conventional staging systems utilized tumor size with some cutoff value to determine tumor stages, Some other studies had suggested a threshold effect of tumor size on long-term outcomes. ${ }^{17,21,22}$ The effect of tumor size on prognosis remains controversial. Nomogram (a simple graphical representation) is an effective and accurate tool to predict long-term prognosis in various cancers. ${ }^{23-25}$ It could incorporate more clinicopathological variables and make full use of some important prognostic factors compared with the conventional staging system. ${ }^{26}$ Yang et al. ${ }^{27}$ has adopted this method accurately predict prognosis in patients with multiple HCCs with concordance index (C-index) of 0.75 . Li et al. ${ }^{28}$ created a nomogram to predict the prognosis of early stage HCCs with C-index of 0.79. Unfortunately, there is a lack of nomogram that can predict prognosis of patients with solitary HCCs hepatectomy.

The aim of this study was to (1) create prognostic nomograms for predicting individual patient with solitary HCC following hepatectomy, and (2) compare the predictive accuracy of this nomograms with the conventional staging systems.

\section{MATERIALS AND METHODS}

\section{Patients}

A total of 538 solitary HCC patients treated with surgery were collected from a prospectively maintained database at West China Hospital from July 1, 2007 to July 31, 2014. The diagnoses of HCC were all histologically confirmed by experienced liver pathologists in West China Hospital. Clinicopathological variables including age, gender, hepatitis B virus surface antigen, liver cirrhosis, alpha-fetoprotein (AFP), tumor size, MVI, major vascular invasion and differentiation were obtained from our database. Major vascular invasion were determined by abdominal computed tomography (CT) scan or magnetic resonance imaging (MRI) or surgical findings. MVI was defined as microscopic tumor invasion identified in small vessels of tumor tissue sample. ${ }^{29}$ The extent of surgery included one segment, two to three segments and >three segments. The inclusion criteria were as follows: (1) solitary HCCs; (2) initially treated by hepatectomy; (3) without extrahepatic metastasis; (4) HCC patients should have Child-Pugh status A, or Child-Pugh status B which can be improved to A. The exclusion criteria were as follows: (1) with other cancers; (2) the major vascular invasion was involving in the trunk of portal vein or the inferior vena cava; (3) HCC with satellite lesions; and (4) incomplete clinicopathological reports and follow-up data. Informed consent for using their data in research was obtained from all patients. This retrospective study was conducted in accordance with the Declaration of Helsinki and was approved by the Ethic Committee of West China Hospital.

\section{Follow-up}

Follow-up data included time and location of HCC recur- rence, treatments after recurrence, survival information and time of follow-up. The median follow-up time was 40.5 months in the training set and 40.2 months in the validation set. All of the patients were regularly followed at first month and every 3 months after surgery. Routing blood tests, liver function tests, AFP levels measurements, HBV-DNA levels, liver ultrasound were performed at each follow-up. Once HCC recurrence was suspected, contrast-enhanced computed tomography, and MRI was performed to confirm the lesions, and chest CT scan and bone scan were applied if necessary. After HCC recurrence, patients were evaluated at multidisciplinary team (MDT) in West China Hospital for treatment guidance based on the status of tumor and general condition. The MDT mainly comprised of experienced hepatic surgeon, radiologists, pathologists and oncologists in West China Hospital. Salvage liver transplantation, resection, radiofrequency ablation (RFA), and transarterial chemoembolization (TACE) and best care support (BSC) could be applied. Patients were administrated antivirus therapy, such as Entecavir (0.5 mg/day), if their HBV-DNA levels were $>1.00 \mathrm{E}+10^{3} \mathrm{IU} / \mathrm{mL}$ before and after surgery during follow-up. Recurrence-free survival (RFS) and overall survival (OS) was calculated from the date of initial treatment until the date of detection of recurrence and death or the date of last follow-up. In the BCLC subgroups, tumor with great than $5 \mathrm{~cm}$ in diameter was classified into BCLC stage B HCC as previous study. The mean value of platelet to lymphocyte ratio (PLR) and neutrophil to lymphocyte ratio (NLR) was 108 and 3.0.

\section{Statistical analysis}

Continuous variables were reported with means \pm standard deviation compared using Student t-test, and categorical variables were presented with frequency and percent compared using the chi-square test. Significant factors in the univariate analysis were included in the multivariate analysis model. Finally, the multivariate Cox proportional hazard model was used to identify independent prognostic factors using the step-forward method. Restricted cubic spline was applied to analyze the relationship between the continuous variables, such as tumor size, and its risk on the prognosis. ${ }^{30}$ Nomogram was established based on results of independent risk factors on multivariable analyses. For nomogram construction and validation, we randomly assigned $80 \%$ of the patients to the training set $(n=419)$ and $20 \%$ to the validation set $(n=119)$.

Model performance was evaluated with respect to discrimination and calibration. For discrimination Harrell's C-index which is appropriate for censored data the ability of a staging system to stratify survival. A staging system with a concordance index of 1 would have predicted the correct outcomes in all patients; a concordance index of 0 would mean that the incorrect outcomes were predicted in all patients; a concordance index of 0.5 would imply that the system correctly predicted the outcomes $50 \%$ of the time. ${ }^{31}$ The bootstrap method was used to obtain 
relatively unbiased estimate (1,000 bootstrap samples). For calibration, we presented the calibration curves for 3- and 5-year survival, which depict the agreement between predicted and the actual observed Kaplan-Meier estimates of survival probability. The same methods, discrimination and calibration, were performed for the validation set. All analyses were performed using $\mathrm{R}$ package with the rms and hmisc. The statistical analysis was carried out using the SPSS 20.0 and R software version 3.3.0 (http://www.r-project.org/). A p-value $<0.05$ was considered as statistically significant.

\section{RESULTS}

1. Clinicopathologic characteristics and follow-up information of patients

A total of 538 patients with solitary HCC who received hepatectomy were randomly divided into the training set (419 patients, 80\%) and validation set (119 patients, 20\%). The clinicopathologic characteristics of patients in the training set and validation set are listed in Table 1 . Of all the patients, 465 patients (86.4\%) had positive HBV infection and 262 patients (48.7\%) had HBV-DNA load $>10^{3} \mathrm{IU} / \mathrm{mL}$. The tumor size was $6.8 \pm 3.7 \mathrm{~cm}$. One hundred seventy-four patients (32.3\%) had presence of MVI and 73 patients (13.6\%) had presence of major vascular invasion. The distribution of these characteristics is almost similar among the training set and validation set. At the end of the follow-up, 293 patients (54.5\%) and 61 patients (11.3\%) suffered from intra-hepatic recurrence and extrahepatic recurrence. Six patients (1.1\%) received salvage liver transplantation therapy, 36 patients (6.7\%) received re-resection, 31 patients (5.8\%) received RFA, 129 patients (24.0\%) received TACE and 152 patients (28.3\%) received BSC. The median survival for all patients was 56 months (95\% confidence interval [CI], 47.7 to 64.2 months). The 1-, 3-, and 5-year OS was 84.5\%, $61.7 \%$, and $46.5 \%$, respectively.

\section{Predictors of survival (training set)}

The results of the univariate and multivariate analyses are presented in Table 2. From the univariate analysis, gender, AFP, aspartate aminotransferase, platelet, PLR, NLR, tumor size, MVI, major vascular invasion and the extent of surgery were associated with survival. These variables were selected for the multivariate model. Finally, tumor size $(\mathrm{p}<0.001$; hazard ratio [HR], 1.114; 95\% CI, 1.077 to 1.153), MVI ( $\mathrm{p}=0.002$; HR, 1.614; $95 \%$ CI, 1.185 to 2.197$)$ and major vascular invasion ( $\mathrm{p}=0.002$; HR, 3.913; 95\% CI, 2.717 to 5.636) were significantly associated with survival (Table 2). The effect of continuous variable (tumor size) was explored using restricted cubic splines. Tumor size had nonlinear effects on the prognosis. We noted that the effect of tumor size on the HR of prognosis was linear below and above a threshold of approximately $7 \mathrm{~cm}$ (Fig. 1).
Table 1. Patient Characteristics and Follow-up Information

\begin{tabular}{|c|c|c|}
\hline Variable & $\begin{array}{l}\text { Training set } \\
(\mathrm{n}=419)\end{array}$ & $\begin{array}{c}\text { Validation set } \\
\quad(\mathrm{n}=119)\end{array}$ \\
\hline \multicolumn{3}{|l|}{ Age, yr } \\
\hline$>60$ & 93 (22.2) & 39 (32.8) \\
\hline$\leq 60$ & $326(77.8)$ & $80(67.2)$ \\
\hline \multicolumn{3}{|l|}{ Sex } \\
\hline Male & 368 (87.8) & $97(81.5)$ \\
\hline Female & $51(12.2)$ & $22(18.5)$ \\
\hline Positive HBsAg & 366 (87.4) & 99 (83.2) \\
\hline HBV-DNA $>10^{3}$ copies $/ \mathrm{mL}$ & $206(49.2)$ & $56(47.1)$ \\
\hline \multicolumn{3}{|l|}{ AFP, ng/mL } \\
\hline$>400$ & $191(45.6)$ & 47 (39.5) \\
\hline$\leq 400$ & $228(54.4)$ & $72(60.5)$ \\
\hline TBIL, $\mu \mathrm{mol} / \mathrm{L}$ & $14.9 \pm 6.1$ & $15.7 \pm 6.8$ \\
\hline AST, U/L & $51.4 \pm 37.9$ & $55.1 \pm 39.8$ \\
\hline ALT, U/L & $57.3 \pm 57.0$ & $61.3 \pm 61.7$ \\
\hline $\mathrm{N}$ & $14.9 \pm 6.1$ & $15.7 \pm 6.8$ \\
\hline $\mathrm{L}$ & $1.5 \pm 1.1$ & $1.4 \pm 0.5$ \\
\hline PLT, $\times 10^{9} / \mathrm{L}$ & $143.2 \pm 67.7$ & $141.1 \pm 69.4$ \\
\hline \multicolumn{3}{|l|}{ PLR } \\
\hline$>108$ & $154(36.8)$ & $40(33.6)$ \\
\hline$\leq 108$ & $265(63.2)$ & $79(66.4)$ \\
\hline \multicolumn{3}{|l|}{ NLR } \\
\hline$>3.0$ & $115(27.4)$ & 38 (31.9) \\
\hline$\leq 3.0$ & 304 (72.6) & $81(68.1)$ \\
\hline Tumor size, cm & $6.7 \pm 3.7$ & $6.7 \pm 3.5$ \\
\hline \multicolumn{3}{|l|}{ Differentiation } \\
\hline Poor & $170(40.6)$ & $52(43.7)$ \\
\hline Moderate-well & $249(59.4)$ & $67(56.3)$ \\
\hline MVI & $135(32.2)$ & 39 (32.8) \\
\hline Major vascular invasion & $66(15.8)$ & $7(5.9)$ \\
\hline Cirrhosis & $265(63.2)$ & $72(60.5)$ \\
\hline ALB, g/dL & $41.4 \pm 4.5$ & $41.2 \pm 4.1$ \\
\hline Blood transfusion & $40(9.5)$ & $6(5.0)$ \\
\hline \multicolumn{3}{|l|}{ The extent of surgery } \\
\hline >3 Segments & $93(22.2)$ & $26(21.8)$ \\
\hline 2-3 Segment & $127(30.3)$ & $42(35.3)$ \\
\hline 1 Segment & $199(47.5)$ & $51(42.9)$ \\
\hline \multicolumn{3}{|l|}{ Recurrence } \\
\hline Intrahepatic & $227(54.1)$ & $66(55.5)$ \\
\hline Extrahepatic & $50(11.9)$ & $11(9.2)$ \\
\hline
\end{tabular}

Data are presented as number (\%) or mean \pm SD.

$\mathrm{HBsAg}$, hepatitis B virus surface antigen; HBV-DNA, hepatitis B viral-DNA; AFP, $\alpha$-fetoprotein; TBIL, total bilirubin; AST, aspartate aminotransferase; ALT, alanine aminotransferase; N, neutrophil; L, lymphocyte; PLT, platelet; PLR, platelet to lymphocyte ratio; NLR, neutrophil to lymphocyte ratio; MVI, microvascular invasion; ALB, albumin. 
Table 2. Variables Associated with Overall Survival According to the Cox Proportional Hazards Model

\begin{tabular}{|c|c|c|c|c|}
\hline \multirow{2}{*}{ Variable } & \multicolumn{2}{|c|}{ Univariate analysis } & \multicolumn{2}{|c|}{ Multivariate analysis } \\
\hline & $\mathrm{p}$-value & HR (95\% CI) & p-value & HR (95\% CI) \\
\hline Age $(>60 / \leq 60)$ & 0.072 & & & \\
\hline Sex (male/female) & 0.026 & $1.769(1.071-2.893)$ & & \\
\hline Positive HBsAg & 0.791 & & & \\
\hline HBV-DNA & 0.181 & & & \\
\hline AFP $(>400 / \leq 400)$ & $<0.001$ & $1.683(1.275-2.223)$ & & \\
\hline TBIL & 0.552 & & & \\
\hline AST & 0.008 & $1.004(1.001-1.007)$ & & \\
\hline ALT & 0.704 & & & \\
\hline $\mathrm{N}$ & 0.245 & & & \\
\hline $\mathrm{L}$ & 0.702 & & & \\
\hline PLT & 0.001 & $1.003(1.001-1.005)$ & & \\
\hline $\operatorname{PLR}(>108 / \leq 108)$ & 0.004 & $1.525(1.148-2.027)$ & & \\
\hline NLR $(>3.0 / \leq 3.0)$ & $<0.001$ & $1.793(1.337-2.405)$ & & \\
\hline Tumor size & $<0.001$ & $1.139(1.105-1.175)$ & $<0.001$ & $1.114(1.077-1.153)$ \\
\hline Differentiation (poor/moderate-well) & 0.154 & & & \\
\hline MVI & $<0.001$ & $2.599(1.958-3.449)$ & 0.002 & $1.614(1.185-2.197)$ \\
\hline Major vascular invasion & $<0.001$ & $5.969(4.232-8.420)$ & 0.002 & $3.913(2.717-5.636)$ \\
\hline Cirrhosis & 0.641 & & & \\
\hline ALB & 0.391 & & & \\
\hline Blood transfusion & 0.062 & & & \\
\hline The extent of surgery & $<0.001$ & 1.629 (1.376-1.928) & & \\
\hline
\end{tabular}

$\mathrm{HR}$, hazard ratio; CI, confidence interval; HBsAg, hepatitis B virus surface antigen; HBV-DNA, hepatitis B viral-DNA; AFP, $\alpha$-fetoprotein; TBIL, total bilirubin; AST, aspartate aminotransferase; ALT, alanine aminotransferase; N, neutrophil; L, lymphocyte; PLT, platelet; PLR, platelet to lymphocyte ratio; NLR, neutrophil to lymphocyte ratio; MVI, microvascular invasion; ALB, albumin.

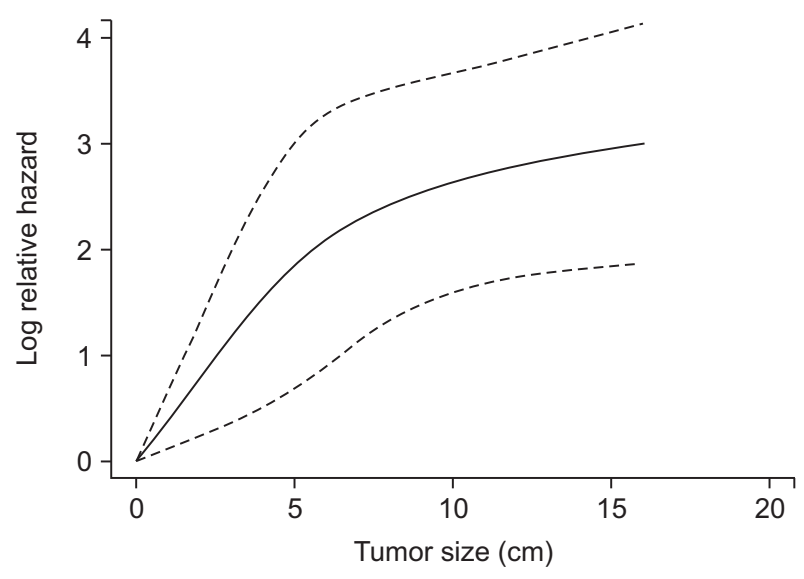

Fig. 1. The effect of increasing tumor size on prognosis in a Cox analysis using restricted cubic splines. At the cutoff value of $7 \mathrm{~cm}$, the hazard ratio for the prognosis changed. Solid line indicates the mean value. Dotted line indicates the 95\% confidence interval.

\section{Construction and validation of nomograms}

Variables included in the multivariate model were further utilized to construct the nomogram (Fig. 2A). Based on the nomogram, we could obtain an estimate of the probability of 3- and 5-year survival rate for solitary HCC following hepatectomy. Patient with a higher score had a worse prognosis. The Cindex values in the training set were 0.75 (95\% CI, 0.72 to 0.78) for OS. The calibration curves showed good agreement between predicted and observed outcomes for the 3-and 5-year OS (Fig. $3 \mathrm{~A}$ and $\mathrm{B}$ ). In the validation set, this OS nomogram was assessed with a C-index was 0.78 (95\% CI, 0.72 to 0.84). The calibration curves also showed good agreement between predicted and observed outcomes for the 3-and 5-year OS (Fig. 3C and D).

Similarly, tumor size, MVI and major vascular invasion were used to generate the RFS nomogram (Fig. 2B). The C-index values in the training set were 0.72 (95\% CI, 0.69 to 0.75$)$. In the validation set, The C-index was 0.78 ( $95 \%$ CI, 0.72 to 0.84 ).

\section{OS comparison of between nomogram and conventional staging systems}

We compared the discrimination of the nomogram with that of conventional staging system. The training data set C-index was 0.75 . However, the C-index was 0.71 for BCLC staging system ( $p=0.003), 0.66$ for the seventh AJCC edition ( $p<0.001), 0.68$ for CLIP $(p<0.001)$ and 0.70 for HKLC $(p<0.001)$ which means 


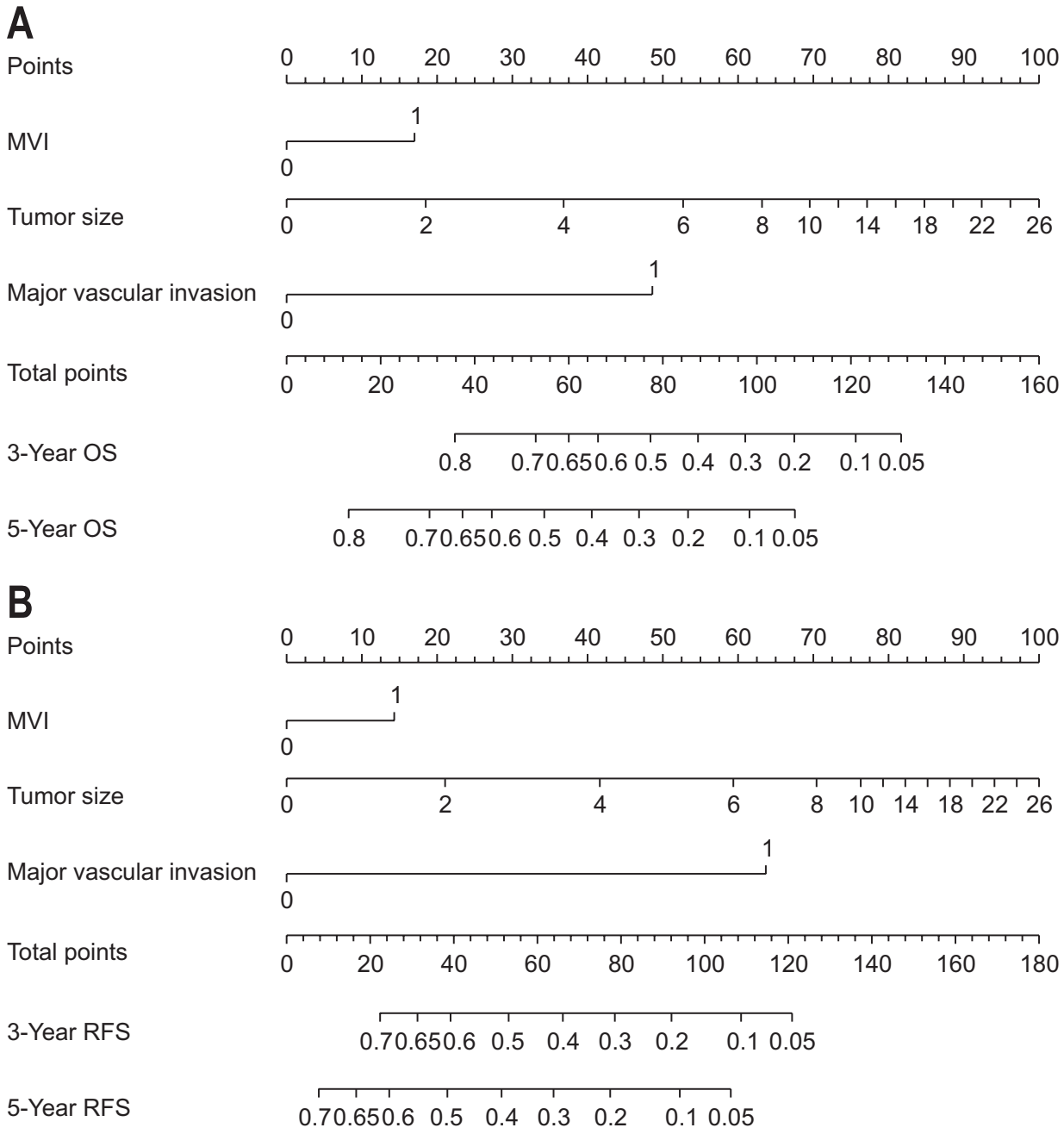

Fig. 2. Nomograms for predicting overall survival (OS) (A) and recurrence-free survival (RFS) (B) in hepatocellular carcinoma (HCC) patients after hepatectomy. For each predictor, a straight upward line is drawn to determine the points. The cumulative points are plotted on the total points bar, and a straight downward line shows the 3- and 5-year estimated postoperative survival rates. Microvascular invasion (MVI): 0, none; 1, presence of MVI; Major vascular invasion: 0, none; 1 , presence of Major vascular invasion. low capability of discrimination and concordance between the estimated and the actual events.

The validation set C-index was 0.78. However, the C-index was 0.69 for BCLC staging system $(\mathrm{p}<0.001), 0.66$ for the seventh AJCC edition $(p<0.001), 0.71$ for CLIP $(p=0.024)$ and 0.70 for HKLC $(p<0.001)$ which means lower capability of discrimination and concordance between the estimated and the actual events. The results suggest that the nomogram was a useful predictor for survival of patients with solitary HCC in the training set and validation set (Fig. 4).

\section{DISCUSSION}

In this study, we describe nomograms which accurately predict an individual's prognosis of solitary HCCs following hepatectomy. Tumor-related variables were the most important predictors after curative surgery in our study. The status of liver cirrhosis had less impact on the prognosis in our study. Finally, our nomograms consisted of three simple parameters (tumor size, MVI, and major vascular invasion). The study had utilized a large cohort of patients to derive and validate the nomogram.
The nomogram performed well, with good discriminatory prognostic ability, and therefore may be helpful to individualized post-operative treatment decisions and counseling. The nomogram had advantages over the conventional staging systems, because it could fully take into account predictors to provide prognostic information. Despite its widespread use worldwide in HCCs, this is the first to create predictive nomogram for heterogeneously solitary HCCs.

Solitary HCCs consist of a heterogenous group. In our study, tumor size varied greatly with a median of $6.7 \pm 3.7 \mathrm{~cm}$ ranging from 1.2 to $25.0 \mathrm{~cm}$. There were 174 patients (32.3\%) with MVI and 73 patients (13.6\%) with major vascular invasion. The 3and 5-year survival rate was $65.5 \%$ and $48.8 \%$ after surgery for solitary HCCs.

The impacts of tumor size on prognosis remain controversial. Some investigators considered that for solitary HCCs, increasing tumor size did not decrease the prognosis after surgery. ${ }^{9,19}$ Besides, the current BCLC staging system classified single HCC into stage A HCC regardless of tumor size. ${ }^{3}$ However, some other investigators held the oppose opinion and suggested that solitary large HCC $(>5 \mathrm{~cm}$ ) should be classified as BCLC stage B 
HCCs. ${ }^{18,32,33}$ Most investigators identified that over a threshold of tumor size, such as $7 \mathrm{~cm}, 8 \mathrm{~cm}$ or $5 \mathrm{~cm}$, had negative impacts on the prognosis. ${ }^{8,1734}$ Even some investigator proposed that patients with solitary HCC should be subclassified based on tumor size. ${ }^{35}$ In this study, tumor size (HR, 1.114) was validated as an independent risk factor associated with the prognosis after curative surgery. Notably, the prognostic implication of tumor size was proven to have a nonlinear effect on prognosis. It had linear effect on the risk of death bellow or above the tumor size appropriate $7 \mathrm{~cm}$. The score of tumor size equal to $26 \mathrm{~cm}$ were 100 points. Unlike the conventional staging systems, this nomogram incorporated the tumor size as continuous variables. The larger was the tumor, the higher points were the score.

In addition to tumor size, vascular invasion were strong predictors for the prognosis after liver resection based on multivariate analyses in current study. Consistently, vascular invasion had a strong effect on long-term survival of small or large single HCC and promoted distant metastasis. ${ }^{10,18,36}$ Even some investigators advocated the advantage of MVI over the Milan criteria in stratifying the prognosis. ${ }^{12}$ Except for the seventh

\section{A}

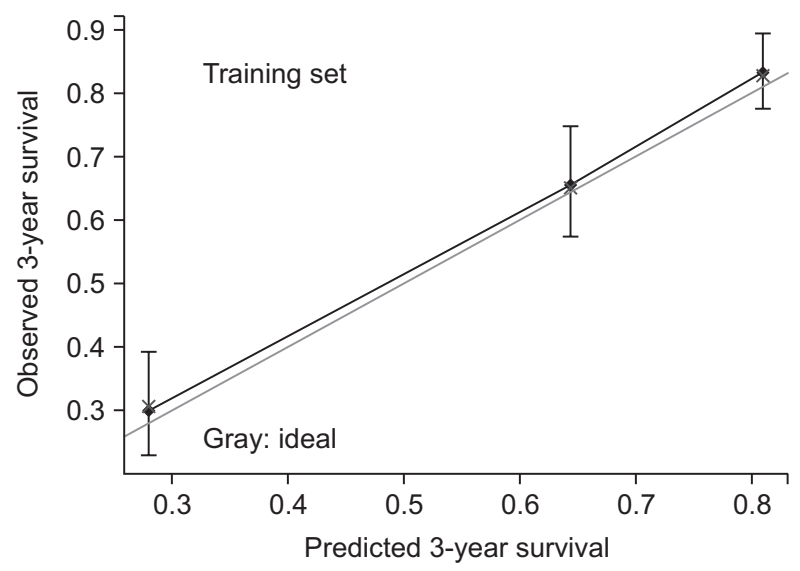

C

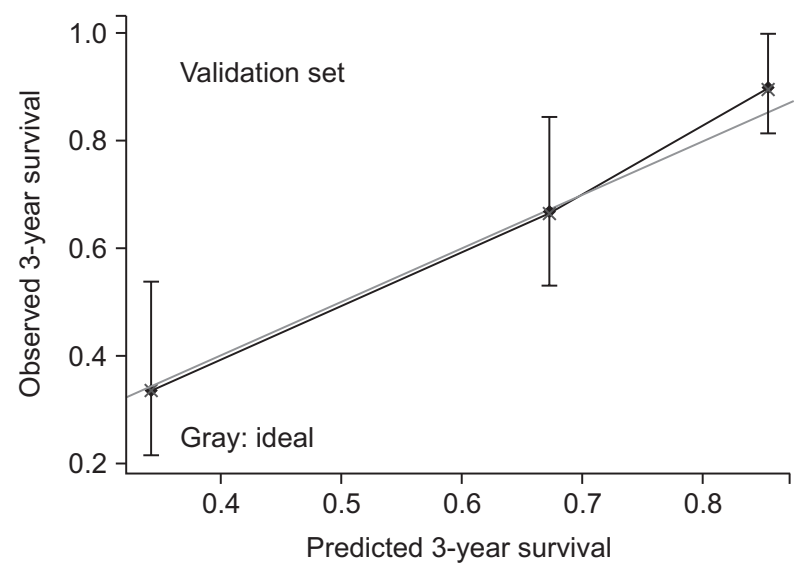

AJCC, few staging systems incorporate the MVI when classifying the tumor stage. In this nomogram, the presence of MVI was assigned 17 points. Undoubtedly, the presence of major vascular invasion was strongly associated with a decreased survival probability, which was assigned a score as high as 48.6 points.

The conventional staging systems could place the patients in different prognostic groups. However, our nomogram can predict individual prognosis after hepatectomy. The conventional staging system might perform well in stratifying the prognosis. The seventh AJCC and BCLC staging system showed good prognostic stratification for solitary HCCs in the training set and validation set. However, in the training set, the HKLC was unsatisfactory in stratifying patients between stages III and IV ( $p=0.390)$, and the CLIP was unsatisfactory in stratifying patients between stages II and III ( $p=0.629)$. In the validation set, CLIP was unsatisfactory in stratifying patients between stages II and III ( $\mathrm{p}=0.197)$, and stages III and IV ( $\mathrm{p}=0.054)$.

Compared with the BCLC staging system, the CLIP and HKLC staging system, this nomogram incorporated the MVI when calculated the risk points. Compared with the seventh AJCC, this
B

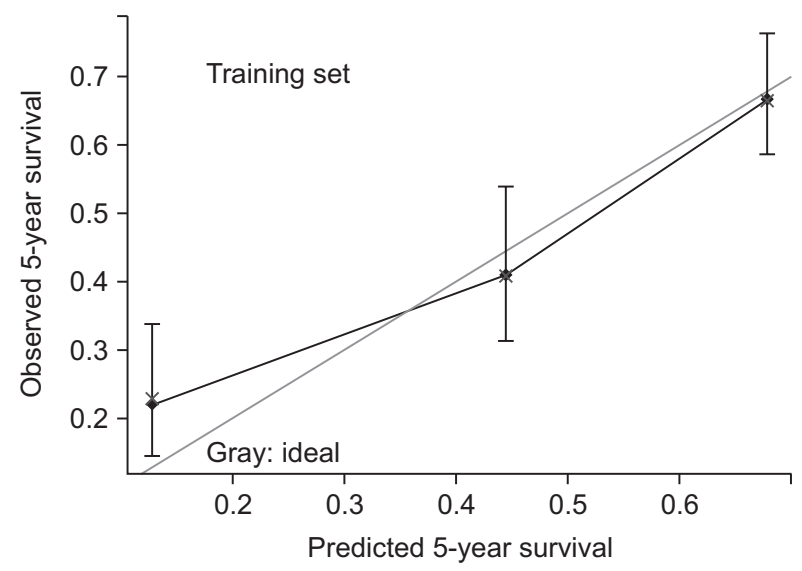

D

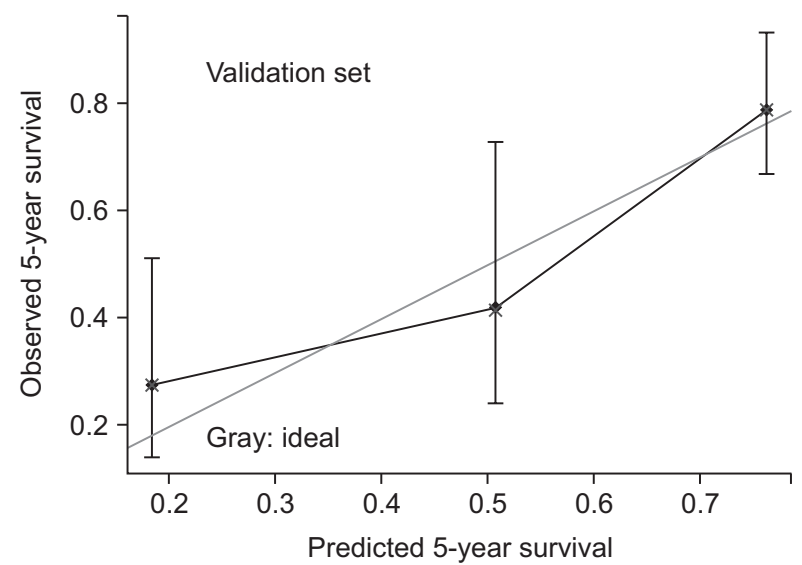

Fig. 3. Calibration plots of 3- and 5-year survival rates for the training set (A, B) and validation set (C, D). Nomogram-predicted probability of overall survival is plotted on the X-axis; actual overall survival is plotted on the Y-axis. The gray line indicates the ideal nomogram reference line. Vertical bars represent 95\% confidence intervals. 
nomogram incorporated the tumor size as continuous variable which had nonlinear effect on the prognosis. Moreover, the limitation of the current AJCC for solitary HCCs has been reported in previous studies in term of tumor size. ${ }^{37,38}$ Taken together, our nomogram for solitary HCCs quantified the patients' risk using the most important predictors in flexible way. When compared with the four staging systems, our nomogram showed better discriminatory power for OS of solitary HCCs with the C-index ( 0.75 and 0.78 for the training and validation set, respectively). Our RFS nomogram had index of 0.72 in the training set and 0.78 for the validation set, respectively. The calibration curve suggested that the gap between observed and nomogram predicted
A

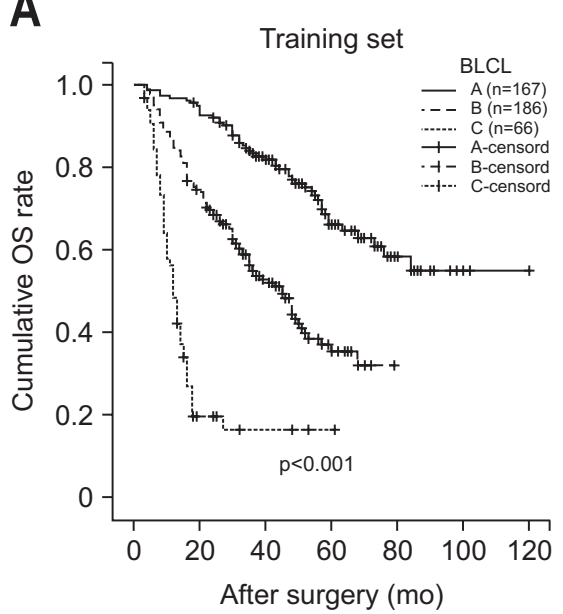

D

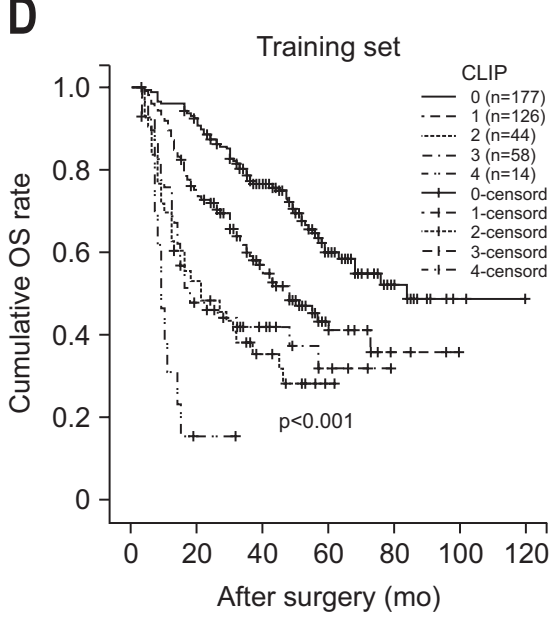

G

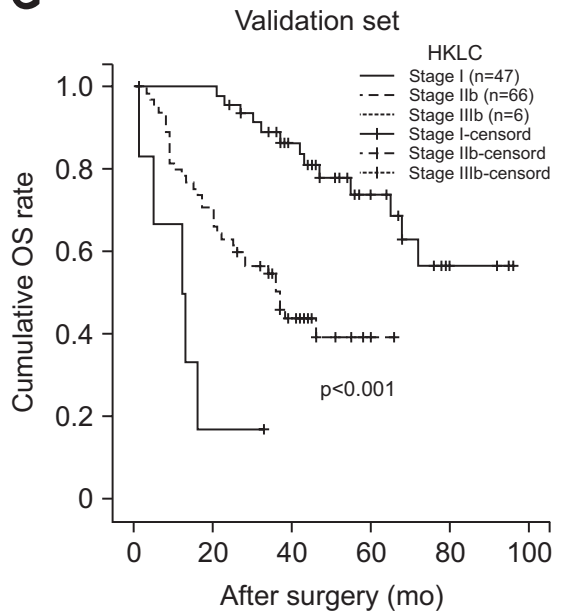

B

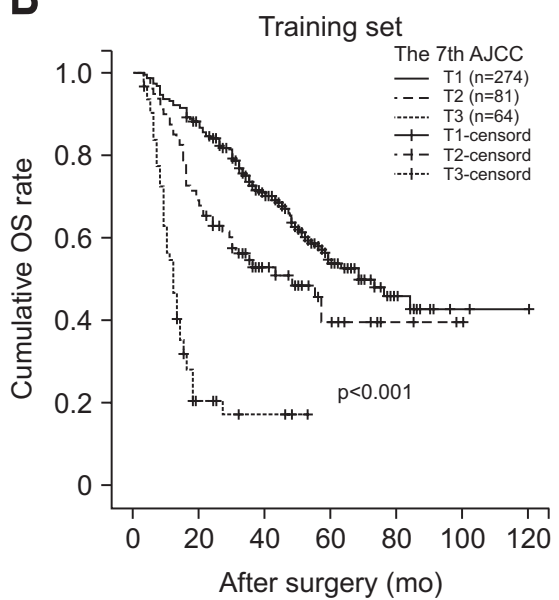

E

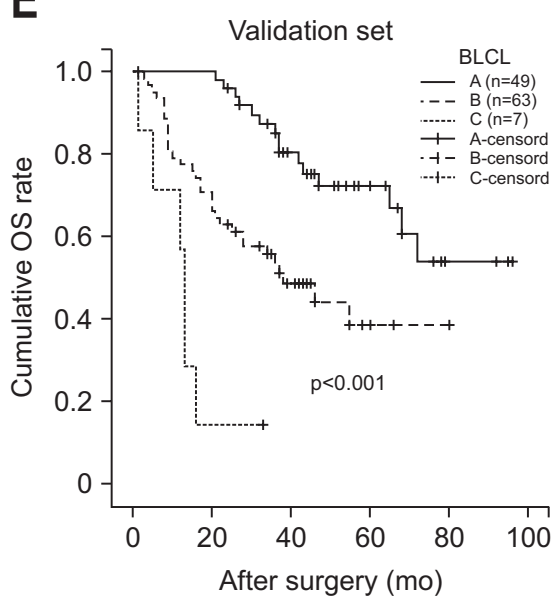

H

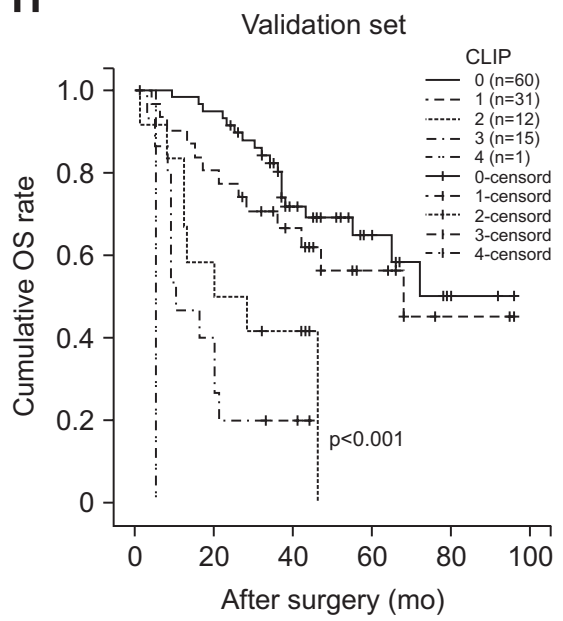

C

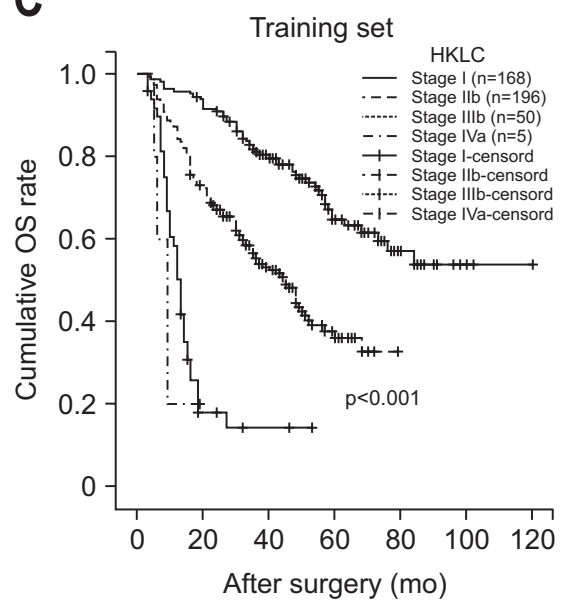

F

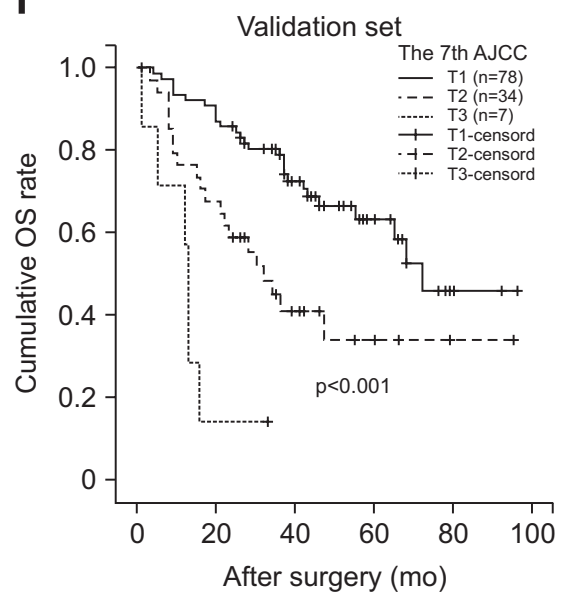

Fig. 4. Kaplan-Meier survival curves of the training and validation sets stratified by the BCLC staging system (A, B), the AJCC seventh edition (C, D), CLIP (E, F), and HKLC (G, H). OS, overall survival; BCLC, Barcelona Clinic Liver Cancer; AJCC, American Joint Committee on Cancer; CLIL, Cancer of the Liver Italian Program; KHLC, Hong Kong Liver Cancer. 
3- and 5-year survival was small in the training set and validation set (Fig. 3). The higher was the total points, the worse is the prognosis.

This study has some limitations. Firstly, this was a retrospective study and thus it needed prospective validation. In addition, external validation from other institutions may facilitate wider use of this prognostic nomogram. Secondly, in the future, molecular studies might identify more risk factors associated with the prognosis and incorporation of these factors might further improve the accuracy of the nomogram. Thirdly, among all HCCs, solitary HCC was the most common cancer type (about $54.8 \%$ ). However, the proportion of solitary HCC patients who underwent surgical resection is even more limited when considering hepatic function due to cirrhosis. Therefore, the usefulness of this nomogram in real clinical practice may be limited.

In conclusions, our nomograms improve the ability to predict individual patient survival compared with the conventional staging systems. To the best of our knowledge, it is the first nomogram developed for solitary HCCs after surgery in Asia. Using our nomograms to predict 3- and 5-year survival may provide a great benefit in clinical practice.

\section{CONFLICTS OF INTEREST}

No potential conflict of interest relevant to this article was reported.

\section{ACKNOWLEDGEMENTS}

This work was supported by grants from Scientific and Technological Support Project of Sichuan Province (2015SZ0049 and 2016SZ0025).

Author contributions: T.W., proposed the study; J.S., C.Lu, W.C. and C.Li, performed the research; J.S. and L.H., collected and analyzed the data; J.S., wrote the first draft; T.W., reviewed the paper. All authors contributed to the interpretation of the study.

\section{REFERENCES}

1. Wang FS, Fan JG, Zhang Z, Gao B, Wang HY. The global burden of liver disease: the major impact of China. Hepatology 2014;60:2099-2108.

2. Jemal A, Bray F, Center MM, Ferlay J, Ward E, Forman D. Global cancer statistics. CA Cancer J Clin 2011;61:69-90.

3. Bruix J, Han KH, Gores G, Llovet JM, Mazzaferro V. Liver cancer: approaching a personalized care. J Hepatol 2015;62(1 Suppl): S144-S156.

4. Minagawa M, Ikai I, Matsuyama Y, Yamaoka Y, Makuuchi M. Staging of hepatocellular carcinoma: assessment of the Japanese TNM and AJCC/UICC TNM systems in a cohort of 13,772 patients in Japan. Ann Surg 2007;245:909-922.
5. Yau T, Tang VY, Yao TJ, Fan ST, Lo CM, Poon RT. Development of Hong Kong Liver Cancer staging system with treatment stratification for patients with hepatocellular carcinoma. Gastroenterology 2014;146:1691-1700.e3.

6. Lu XY, Xi T, Lau WY, et al. Pathobiological features of small hepatocellular carcinoma: correlation between tumor size and biological behavior. J Cancer Res Clin Oncol 2011;137:567-575.

7. Pawlik TM, Delman KA, Vauthey JN, et al. Tumor size predicts vascular invasion and histologic grade: Implications for selection of surgical treatment for hepatocellular carcinoma. Liver Transpl 2005;11:1086-1092.

8. Lim C, Mise Y, Sakamoto Y, et al. Above $5 \mathrm{~cm}$, size does not matter anymore in patients with hepatocellular carcinoma. World $\mathrm{J}$ Surg 2014;38:2910-2918.

9. Yang LY, Fang F, Ou DP, Wu W, Zeng ZJ, Wu F. Solitary large hepatocellular carcinoma: a specific subtype of hepatocellular carcinoma with good outcome after hepatic resection. Ann Surg 2009;249:118-123.

10. Cai MY, Wang FW, Li CP, et al. Prognostic factors affecting postoperative survival of patients with solitary small hepatocellular carcinoma. Chin J Cancer 2016;35:80.

11. Roayaie S, Jibara G, Taouli B, Schwartz M. Resection of hepatocellular carcinoma with macroscopic vascular invasion. Ann Surg Oncol 2013;20:3754-3760.

12. Lim KC, Chow PK, Allen JC, et al. Microvascular invasion is a better predictor of tumor recurrence and overall survival following surgical resection for hepatocellular carcinoma compared to the Milan criteria. Ann Surg 2011;254:108-113.

13. Zhong JH, Ke Y, Gong WF, et al. Hepatic resection associated with good survival for selected patients with intermediate and advanced-stage hepatocellular carcinoma. Ann Surg 2014;260:329340.

14. Kokudo T, Hasegawa K, Yamamoto S, et al. Surgical treatment of hepatocellular carcinoma associated with hepatic vein tumor thrombosis. J Hepatol 2014;61:583-588.

15. Zhu SL, Ke Y, Peng YC, et al. Comparison of long-term survival of patients with solitary large hepatocellular carcinoma of BCLC stage A after liver resection or transarterial chemoembolization: a propensity score analysis. PLoS One 2014;9:e115834.

16. Pandey D, Lee KH, Wai CT, Wagholikar G, Tan KC. Long term outcome and prognostic factors for large hepatocellular carcinoma (10 cm or more) after surgical resection. Ann Surg Oncol 2007;14:2817-2823.

17. Truant S, Boleslawski E, Duhamel A, et al. Tumor size of hepatocellular carcinoma in noncirrhotic liver: a controversial predictive factor for outcome after resection. Eur J Surg Oncol 2012;38:11891196.

18. Hwang S, Lee YJ, Kim KH, et al. The impact of tumor size on long-term survival outcomes after resection of solitary hepatocellular carcinoma: single-institution experience with 2558 patients. J Gastrointest Surg 2015;19:1281-1290.

19. Zhang H, Yuan SX, Dai SY, et al. Tumor size does not indepen- 
dently affect long-term survival after curative resection of solitary hepatocellular carcinoma without macroscopic vascular invasion. World J Surg 2014;38:947-957.

20. Liu PH, Hsu CY, Hsia CY, et al. Prognosis of hepatocellular carcinoma: assessment of eleven staging systems. J Hepatol 2016;64: 601-608.

21. Tsoulfas G, Mekras A, Agorastou P, Kiskinis D. Surgical treatment for large hepatocellular carcinoma: does size matter? ANZ J Surg 2012;82:510-517.

22. Ansari D, Nordqvist G, Hammarlund P, Dankiewicz J, Massoud A, Andersson R. Solitary liver lesions of indeterminate malignancy: outcome following surgical resection. Anticancer Res 2012;32:3961-3964.

23. Kim Y, Spolverato G, Ejaz A, et al. A nomogram to predict overall survival and disease-free survival after curative resection of gastric adenocarcinoma. Ann Surg Oncol 2015;22:1828-1835.

24. Kent MS, Mandrekar SJ, Landreneau R, et al. A Nomogram to predict recurrence and survival of high-risk patients undergoing sublobar resection for lung cancer: an analysis of a multicenter prospective study (ACOSOG Z4032). Ann Thorac Surg 2016;102:239246.

25. Seisen T, Colin P, Hupertan V, et al. Postoperative nomogram to predict cancer-specific survival after radical nephroureterectomy in patients with localised and/or locally advanced upper tract urothelial carcinoma without metastasis. BJU Int 2014;114:733-740.

26. Vickers AJ, Kattan MW, Daniel S. Method for evaluating prediction models that apply the results of randomized trials to individual patients. Trials 2007;8:14.

27. Yang P, Qiu J, Li J, et al. Nomograms for pre- and postoperative prediction of long-term survival for patients who underwent hepatectomy for multiple hepatocellular carcinomas. Ann Surg 2016;263:778-786.

28. Li J, Zhou J, Yang PH, et al. Nomograms for survival prediction in patients undergoing liver resection for hepatitis B virus related early stage hepatocellular carcinoma. Eur J Cancer 2016;62:86-95.

29. Sumie S, Kuromatsu R, Okuda K, et al. Microvascular invasion in patients with hepatocellular carcinoma and its predictable clinicopathological factors. Ann Surg Oncol 2008;15:1375-1382.

30. Hyder O, Marques H, Pulitano C, et al. A nomogram to predict long-term survival after resection for intrahepatic cholangiocarcinoma: an Eastern and Western experience. JAMA Surg 2014;149: 432-438.

31. Hsiao W, Herrel LA, Yu C, et al. Nomograms incorporating serum C-reactive protein effectively predict mortality before and after surgical treatment of renal cell carcinoma. Int J Urol 2015;22:264270.

32. Jung YK, Jung CH, Seo YS, et al. BCLC stage B is a better designation for single large hepatocellular carcinoma than BCLC stage A. J Gastroenterol Hepatol 2016;31:467-474.

33. Yang J, Li C, Wen TF, Yan LN, Li B, Wang WT, Yang JY, Xu MQ. Is hepatectomy for huge hepatocellular carcinoma $(\geq 10 \mathrm{~cm}$ in diameter) safe and effective? A single-center experience. Asian Pac J Cancer Prev 2014;15:7069-7077.

34. Elnekave E, Erinjeri JP, Brown KT, et al. Long-term outcomes comparing surgery to embolization-ablation for treatment of solitary HCC $<7$ cm. Ann Surg Oncol 2013;20:2881-2886.

35. Liu L, Zhang QS, Pan LH, et al. Subclassification of patients with solitary hepatocellular carcinoma based on post-hepatectomy survival: a large retrospective study. Tumour Biol 2016;37:53275335.

36. Ho CM, Hu RH, Lee PH, Wu YM, Ho MC. Long-term survival in patients with $\mathrm{T} 2$ hepatocellular carcinoma after primary curative resection can be further stratified by tumor size. Medicine (Baltimore) 2014;93:e203.

37. Huang WJ, Jeng YM, Lai HS, Sheu FY, Lai PL, Yuan RH. Tumor size is a major determinant of prognosis of resected stage I hepatocellular carcinoma. Langenbecks Arch Surg 2015;400:725-734.

38. Goh BK, Teo JY, Chan CY, et al. Importance of tumor size as a prognostic factor after partial liver resection for solitary hepatocellular carcinoma: implications on the current AJCC staging system. J Surg Oncol 2016;113:89-93. 